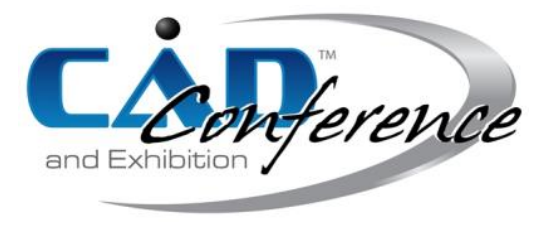

Title:

\title{
A Parking Pose and Trajectory Selection Algorithm Based on Artificial Potential Field and Particle Swarm Optimization
}

Authors:

Wei Huang, hweigo@sjtu.edu.cn, Shanghai Jiao Tong University

Xiangzhi Wei, antonwei@sjtu.edu.cn, Shanghai Jiao Tong University

Jiaye Zhu, jiayezhu@sjtu.edu.cn, Shanghai Jiao Tong University

Kaining Dai, 836526236@sjtu.edu.cn, Shanghai Jiao Tong University

Keywords:

Automated Valet Parking, Collision Detection, Artificial Potential Field, Particle Swarm Optimization, Spline Interpolation

DOI: 10.14733/cadconfP.2021.349-353

Introduction:

Path planning is one of the fundamental issues for automated valet parking system. However, current automatic parking system solutions simply choose the center of the parking bay as target parking pose, which is not the most suitable pose in many situations. Besides, it requires further study to effectively evaluate the parking trajectory; particularly, the path generator might not figure out the suitable trajectory if adjacent vehicle is improperly parked.

This paper presents an optimal parking pose and trajectory selection approach based on the information of the ultrasonic radar and vision. To evaluate the positions of obstacles and parking lines when parking, we construct a virtual potential field that can effectively represent the real parking scenario. Thereafter, particle swarm optimization approach is used to iterate possible parking poses and select the one with the least risk. The parking trajectory is generated and optimized to minimize the risk degree, and finally smoothed using spline interpolation. The experiment results show that our method can adaptively adjust the parking pose in different parking scenarios, and able to generate feasible and smooth parking trajectory from arbitrary starting pose to the optimal parking pose.

\section{Main Sections:}

Artificial Potential Field Construction and Cost Evaluation

Considering the high price of lidar, most vehicles on the market nowadays are using cameras or ultrasonic radar for advanced driver-assistance systems. The camera can obtain the lane line information on the road using lane-line detection algorithm, and the ultrasonic radar can quickly detect physical obstacles such as other vehicles and fences [5]. However, even for some multi-sensor detection schemes equipped with both ultrasonic radars and cameras, only one type of information is used during the auto-parking process, and this is insufficient. In this paper, we propose an artificial potential field method to fuse the information of the ultrasonic radar and vision camera such that the circumstances around the vehicle can be better evaluated.

Artificial potential field is a commonly used path planning algorithm introduced by Khatib [2]. However, considering that parking normally happens in narrow areas, and directly using traditional artificial potential field method often fails to find a feasible solution [4]. Also, the algorithm cannot guarantee that the motion of the vehicle satisfies the kinematic constraints. Therefore, in our approach, a modified artificial potential field method is developed to help evaluate the safety of the 
surrounding environment, and the parking path is defined geometrically and search for the optimal one in configuration space.

Let $W$ denote the 2-D Euclidean parking plane. To distinguish the two different types of obstacles in the neighborhood of the parking space detected by ultrasonic radars and cameras, the following notation is used in this paper [1]. Physical obstacles detected by ultrasonic radar are represented as the compact sets of points $O U_{i}, i=1,2, \ldots, n$ in $W$. Similarly, parking lines detected by the camera are represented as $O C_{i}, i=1,2, \ldots, m$. As shown in Eqn. (1.1), the potential energy $U$ at point $x$ is computed by summing up the effects of all the neighborhood obstacles. For a specific obstacle point $O U_{i}$ or $O C_{i}$, the potential energy it contributes at point $\mathrm{x}$ is computed in Eqn. (1.2).

$$
\begin{gathered}
U(x)=\sum_{j \subset \mathcal{O U}} U_{j}(x)+\sum_{j \subset \mathcal{O C}} U_{j}(x) \\
U_{j}(x)=\left\{\begin{array}{cc}
U_{j \max } & d_{j}(x)=0 \\
0.5 * \eta_{j} *\left(\frac{1}{d_{j}(x)}-\frac{1}{Q_{j}}\right)^{2} & 0<d_{j}(x) \leq Q_{j} \\
0, & d_{j}(x)>Q_{j}
\end{array}\right.
\end{gathered}
$$

With,

$$
U_{\mathcal{O U} \max } \gg U_{\mathcal{O C} \max }, \eta_{\mathcal{O U}} \gg \eta_{\mathcal{O C}}, Q_{\mathcal{O U}} \gg \mathrm{Q}_{\mathcal{O C}}
$$

where $d_{j}(x)$ is the distance from point $x$ to the obstacle $j, \eta_{j}$ is the potential repulsive constant, $Q_{j}$ is the range of the influence of obstacle $j$. During the auto-parking process, the vehicle first needs to ensure that it can avoid physical obstacles, and then try to park as close as possible along the middle of the parking space according to the parking lines. Therefore, for obstacles $O U$ detected by ultrasonic radars, $U_{j \max }, \eta_{j}$, and $Q_{j}$ are assigned with large values to ensure that the vehicle keeps a safe distance from it. For the parking lines $O C$ detected by the cameras, these parameters are assigned with relatively small values. Their relationship is described in Eqn. (1.3). In this way, our algorithm can fuse different obstacle information and evaluate their effects.

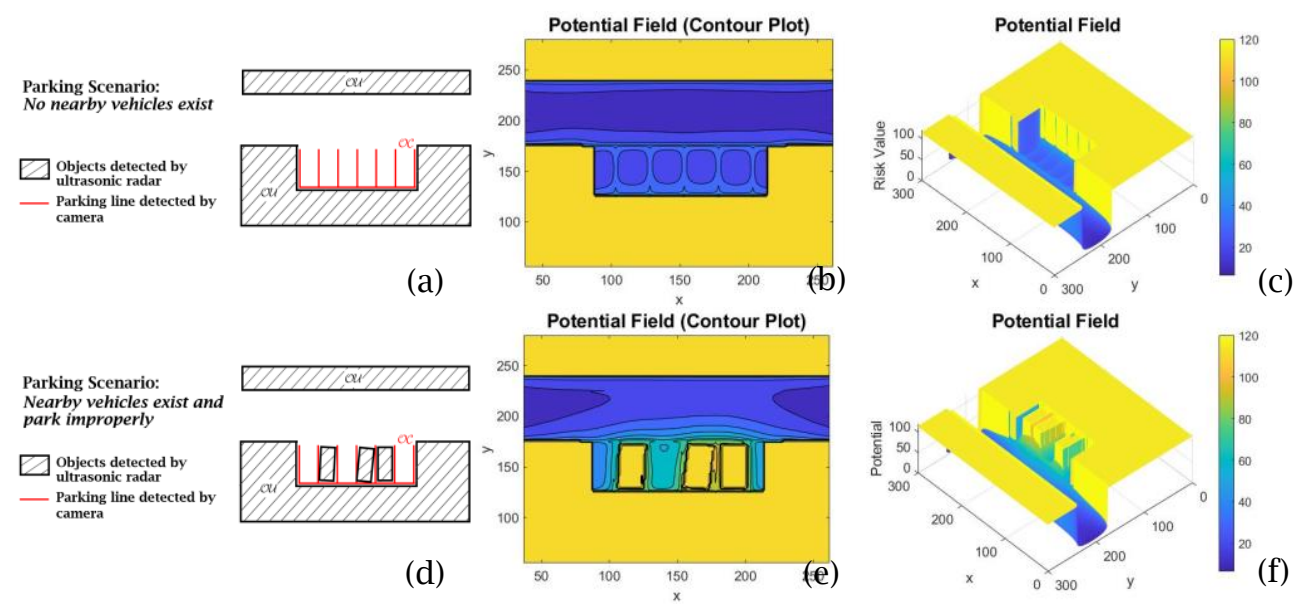

Fig. 1: The first row shows the parking scenario without nearby vehicles: (a) Parking lot map, (b) Potential filed (contour plot), (c) Potential filed. The second row shows the parking scenario when 
nearby vehicles exist and the parking condition is imperfect: (d) Parking lot map, (e) Potential filed (contour plot), (f) Potential filed.

Fig. 1 shows two common parking scenarios in the underground garage and the corresponding potential field. Fig 1. (a) and (d) is the initial parking environment. The dash area denotes the obstacles detected by ultrasonic radars and the red lines denote the parking lines detected by cameras. Fig 1. (b), (c), (e), (f) shows the corresponding potential fields. The higher the potential energy (yellower part in the figure), the more likely the autonomous vehicle collides with obstacles or crosses the lane lines.

\section{Optimal Parking Pose Computation}

To describe the final parking pose of the vehicle, three control parameters are used: vehicle center's coordinate $[x, y]^{\mathrm{T}}$, and the vehicle's heading angle $\theta$ relative to the $\mathrm{x}$-axis shown in Fig 1 . Hence, the pose configuration can be represented as $q=[x, y, \theta]^{\mathrm{T}}$. All possible final parking poses form the configuration space $C$, which is a Euclidean group of $\mathrm{SE}(2)=\mathrm{R}^{2} \times \mathrm{SO}(2)$. Let $A(q)$ be a subset of $C$ referring to the points covered by the body of vehicle at configuration $q$. After constructing the potential field, the risk degree for a given vehicle pose $q$ can be measured by sampling the vehicle coverage space $A(q)$ and computing its average potential energy. Therefore, the problem of selecting the optimal parking pose can be transformed into the problem of finding the vehicle pose that minimizes the average potential energy.

$$
\begin{aligned}
\min _{q \in C} \quad f_{\text {pose }}(q) & =\frac{1}{n} \sum_{p \in A(q)} U(p) \\
\text { s.t. } \quad x_{\min } & \leq x \leq x_{\max } \\
y_{\min } & \leq y \leq y_{\max } \\
\theta_{\min } & \leq \theta \leq \theta_{\max }
\end{aligned}
$$

Where $n$ is the sampling number and $p$ refers to the sample point in the vehicle coverage area $A(q)$. We then use the Particle Swarm Optimization (PSO) to solve the above optimization problem and determine the optimal pose. Within the configuration space $C$, particles are first initialized with an arbitrary pose configuration $q-[x, y, \theta]^{\mathrm{T}}$. The velocity attribute $v_{i}^{t}$ determines the particle's position in the next iteration. During the iteration process, each particle updates its velocity according to its previous velocity and the optimal position of individuals and groups, and thus updates its position based on its previous position and the velocity. The iteration rule is shown as follows:

$$
\begin{gathered}
q_{i}^{t+1}=q_{i}^{t}+v_{i}^{t+1} \\
v_{i}^{t+1}=\omega v_{i}^{t}+c_{1} r_{1} Q_{i}^{t}-q_{i}^{t}+c_{2} r_{2} G^{t}-q_{i}^{t}
\end{gathered}
$$

Where, $q_{i}^{t}$ is the position of the particle $i$ at the $t$ th iteration, and $v_{i}^{t}$ is the velocity of the particle $i$ at the $t$ th iteration. After the iteration, the optimal parking pose with minimum potential energy can be obtained. As Fig 2 shows, we test our approach in three common parking scenarios, one ideal parking scenario, and two less perfect parking scenarios. Refer to Fig 2. (a), case 1 shows the ideal scenario when no adjacent vehicles exist $t$, and the parking process is only guided by parking lane markers. It can be seen that our algorithm chooses the parking pose almost at the center of the target parking bay with zero heading angle as expected. Refer to Fig 2. (b), case 2 is the case when two vehicles are already parked on both sides, but too close to the right parking lane. Refer to Fig 2. (c), case 3 is when the nearby vehicles' heading is not perpendicular to the parking spot. As for the last two cases, our method adaptively adjusts the pose slightly to better fit the actual parking condition. Table 1 compares the optimal pose computed by our method with two other parking poses that are default chosen by many automated valet parking systems in case 3 (Fig. 2(c)). The result shows that our algorithm is capable to adaptively adjust the vehicle pose even in imperfect parking scenarios and reach the least average potential energy. 

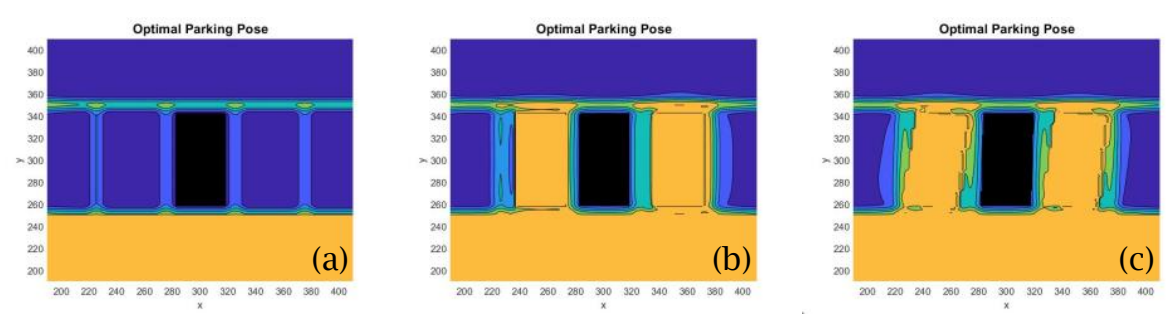

Fig. 2: Optimal parking position. (a) Parking lot without adjacent vehicles, (b) Enclosed parking lot with nearby vehicles parked perfectly, (c) Enclosed parking lot with nearby vehicles parked improperly.

\begin{tabular}{c|cccc}
\hline Parking Pose & $x / m$ & $y / m$ & $\theta /{ }^{\circ}$ & Average Potential Energy \\
\hline Center of parking spot & 0.0 & 0.0 & 90.0 & 20.573 \\
\hline Center of nearby vehicles & 0.0 & 0.0 & 86.0 & 21.066 \\
\hline Our method & $\mathbf{0 . 0 6}$ & $\mathbf{0 . 0 3}$ & $\mathbf{8 8 . 7 8}$ & $\mathbf{2 0 . 3 5 6}$ \\
\hline
\end{tabular}

Tab. 1: Comparison of optimal parking pose with two common default poses in case 3.

\section{Parking Trajectory Computation}

Since the speed is relatively slow during parking, the impact of sliding can be ignored. Therefore, the kinematic model of the autonomous vehicle can be simplified as the Ackermann steering model as shown in Fig 3. (a) [3].
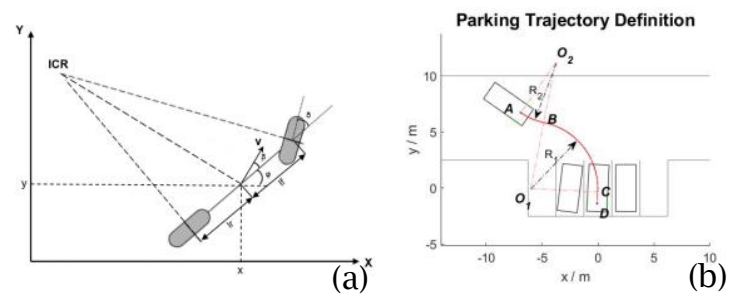

Fig. 3: (a) Ackermann steering model, (b) Parking trajectory definition.

Fig 3. (b) shows the definition of the parking trajectory. Two arcs are used to guide the vehicle from the starting point $\mathrm{A}$ to point $\mathrm{B}$, and point $\mathrm{B}$ to point $\mathrm{C}$, with the turning radius of $R_{2}$ and $R_{1}$, respectively. Then, a line with distance $d$ guides the vehicle to the final parking position. Let $t$ denote a parking trajectory using the above definition, which can be expressed as $t=\left[R_{1}, d\right]^{\mathrm{T}}$. All the possible parking trajectories span a 2-D configuration space and can be represented as $T=t=\left[R_{1}, d\right]^{\mathrm{T}} \mid R_{1} \geq R_{\text {min }}, R_{2} \geq R_{\text {min }}, 0 \leq d \leq d_{\max }$. Here, $R_{\text {min }}$ is the minimum turning radius according to the Ackermann steering model. For a trajectory $t$, we sample several poses along the path, and use the average covered potential energy as a measure of the risk degree of the trajectory. The optimal trajectory selection problem can thus be transformed into an optimization problem as follow.

$$
\begin{aligned}
\min _{t \in T} \quad f_{\text {trajectory }}(t) & =\frac{1}{n} \sum_{q \in t} f_{\text {pose }}(q) \\
\text { s.t. } \quad R_{\min } & \leq R_{1} \\
R_{\min } & \leq R_{2} \\
0 & \leq d \leq d_{\max }
\end{aligned}
$$

Where, 


$$
R_{2}=\frac{\left|A O_{1}\right|^{2}-R_{1}^{2}}{2\left|A O_{1}\right| \cos \angle O_{2} A O_{1}+2 R_{1}}
$$

Next, PSO is also implemented to search for the parking trajectory $t$ with the minimum potential energy in $T$. Since the path generated so far is a composition of circular arcs and line segments, it's discontinuous on the tangent point. This means that the ego vehicle will either needs to be steered or stopped suddenly along the path, which should be avoided. Here, we solve this problem by sampling the trajectory with a given step to obtain discrete points along the path, then use a cubic spline curve to interpolate these points. Fig 4. (a)(b) shows the final parking trajectories our method generated from arbitrary poses to the optimal parking pose in two different parking cases. We also compare our path generator with a widely used approach [3]. The comparison result is shown in Fig 4. (c)(d). It can be seen that our approach selects the final parking pose with a little rotation to better fit in this particular parking scenario. Moreover, our path adjusts the vehicle's heading earlier than the conventional method and does not even cross any side of the parking lane during the parking process.
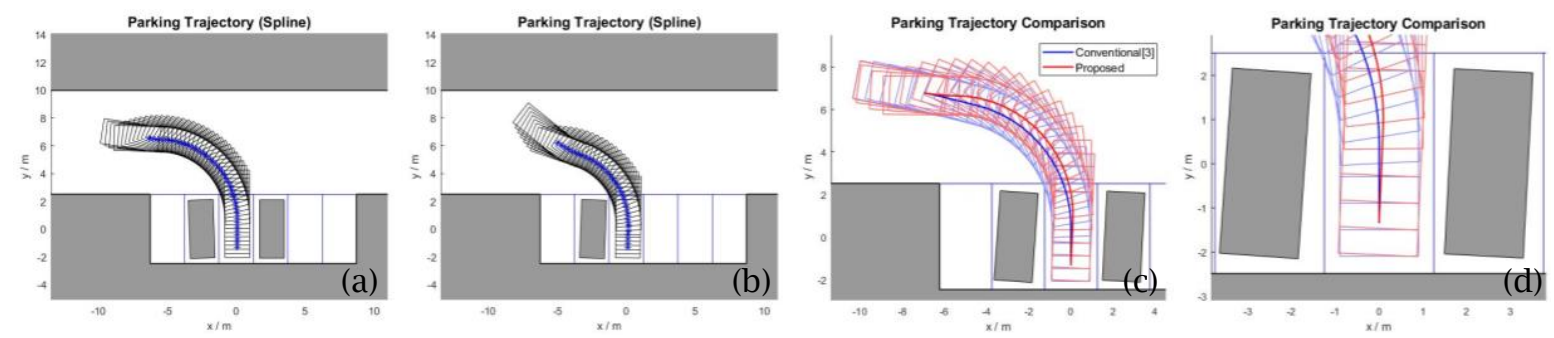

Fig. 4: (a)(b) Final parking trajectory in two different parking scenarios, (c)(d) Trajectory comparison between our method and conventional method, where red lines denote trajectory generated by our method.

\section{Conclusion:}

In this paper, a parking pose selection algorithm based on modified artificial potential field and particle swarm optimization is proposed. The ultrasonic radar and vision information is fused and considered to help select the optimal final parking pose which is most similar to human driver's behavior. We also presented a path-planning algorithm to effectively select a smooth and collisionfree path that minimizes the risk degree. The experimental results demonstrate that our method can adaptively adjust the final parking pose and generate feasible trajectory in different parking scenarios.

\section{References:}

[1] Barraquand, J.; Latombe, J.C.: Robot motion planning: A distributed representation approach, The International Journal of Robotics Research, 10(6), 1991, 628-649. https://doi.org/10.1177/027836499101000604

[2] Khatib, O.: Real-Time Obstacle Avoidance System for Manipulators and Mobile Robots, The International Journal of Robotics Research, 5(1), 1986, 90-98. https://doi.org/10.1177/027836498600500106

[3] Moon, J.; Bae, I.; Cha, J.G.; Kim, S.: October. A trajectory planning method based on forward path generation and backward tracking algorithm for automatic parking systems, In 17th International IEEE Conference on Intelligent Transportation Systems (ITSC), 2017, 719-724. https://doi.org/10.1109/ITSC.2014.6957774

[4] Oetiker, M.B.; Baker, G.P; Guzzella, L.: A navigation-field-based semi-autonomous nonholonomic vehicle-parking assistant, IEEE Transactions on Vehicular Technology, 58(3), 2008, 1106-1118. https://doi.org/10.1109/TVT.2008.928643

[5] Siegwart, R.; Nourbakhsh, I. R.; Scaramuzza, D.: Introduction to autonomous mobile robots, MIT press, 2011. 